REVUE DE L'INSTITUT

FRANÇAIS D'HISTOIRE

EN ALLEMAGNE

\section{Revue de l'IFHA}

Revue de l'Institut français d'histoire en Allemagne

$1 \mid 2009$

IFHA 1

\title{
Sur l'effondrement des Archives Historiques de la ville de Cologne
}

\author{
Klaus Militzer
}

Traducteur : Aurore ARNAUD

URL : http://journals.openedition.org/ifha/342

DOI : 10.4000/ifha.342

ISSN : 2198-8943

Éditeur

IFRA - Institut franco-allemand (sciences historiques et sociales)

\section{Édition imprimée}

Date de publication : 30 septembre 2009

Pagination : 121-125

ISSN : 2190-0078

Référence électronique

Klaus Militzer, «Sur l'effondrement des Archives Historiques de la ville de Cologne », Revue de I'IFHA

[En ligne], 1 | 2009, mis en ligne le 07 février 2013, consulté le 03 mai 2019. URL : http://

journals.openedition.org/ifha/342 ; DOI : 10.4000/ifha.342

Ce document a été généré automatiquement le 3 mai 2019.

(C)IFHA 


\title{
Sur l'effondrement des Archives Historiques de la ville de Cologne
}

\author{
Klaus Militzer \\ Traduction : Aurore ARNAUD
}

Le 3 mars 2009 en milieu de journée, les archives historiques de Cologne se sont effondrées. Depuis et jusqu'à nouvel ordre, les fonds n'en sont plus consultables. Avant ce jour déjà et suite aux travaux de construction du métro sous la rue Séverin devant le bâtiment des archives, une cavité s'était vraisemblablement formée, dans laquelle fut englouti le magasin donnant sur la rue. L'aile administrative, ainsi que les sous-sols dans lesquels étaient conservés les actes officiels, la plupart des photos et la bibliothèque furent à peine touchés. Les actes, les photos, les livres de la bibliothèque et les répertoires ont ainsi pu être mis à l'abri.

Les principales victimes de l'effondrement sont donc avant tout les fonds anciens allant jusqu'à la période dite "française ", jusqu'en 1794 donc, date à laquelle les troupes révolutionnaires françaises sont entrées dans Cologne. Parmi eux figurent les Schreinsbücher, une source unique et de "renommée mondiale», les volumes de parchemin du Grundbuch de la ville de Cologne depuis le XIIe siècle, contenant de nombreuses informations d'importance sur l'histoire sociale, constitutionnelle, commerciale et économique, ainsi que les procès-verbaux du Magistrat et de leurs formes primitives depuis le XIVe siècle, enfin des documents sur les corporations et le commerce. Il y a également les dépôts des monastères et fondations religieuses qui, après avoir été confiés aux archives nationales centrales de Düsseldorf, ont été transférés aux archives de la ville de Cologne après la Seconde Guerre mondiale.

Tout aussi importants que les Schreinsbücher, il y a les documents du fonds « constitution et administration ", dans lequel était conservé le dépôt de l'administration de la ville ancienne; ceux du fonds "Cologne et l'Empire", où se trouvaient essentiellement les documents sur les relations entre Cologne et le Saint-Empire; ceux aussi du fonds «Cologne contre Cologne » où se trouvaient les documents sur les affrontements entre la ville et l'archevêque de Cologne. Le dépôt de l'ancienne Université de Cologne fondée en 
1394 et fermée en 1798 est également d'importance. Il ne faut pas oublier les nombreux volumes de la section «comptabilité» et les documents de ce qu'on appelle « l'administration des pauvres » (Armenverwaltung), un fonds qui n'était que partiellement dépouillé et dans lequel avait été incorporée la documentation portant sur de nombreuses fondations pieuses. Au XVIIe siècle, les archives du comptoir hanséatique de Bruges et Anvers avaient été transférées à Cologne et étaient depuis conservées aux archives municipales. Des dépôts importants sur la Hanse faisaient par conséquent des fonds des archives municipales. S'ajoutent les volumes du Tribunal de la cité, pour autant qu'ils aient été déposés à Cologne, ainsi que des manuscrits divers comme par exemple deux autographes d'Albert le Grand, des collections de toutes sortes avec des copies de documents perdus, remontant même au Moyen Âge. "L'administration française " constituait un fonds à part entière dans lequel les documents de la période d'occupation française de 1798 à 1814 étaient conservés. Les documents sont en partie en langue française ou alors bilingues. Ce fonds est sans aucun doute important, non seulement pour les recherches sur Cologne mais aussi pour l'histoire de la France, puisque cet épisode constitue une partie de son histoire.

Depuis 1814 se trouvait dans les fonds du maire et du directeur de l'administration municipale ainsi que chez d'autres responsables administratifs le dépôt de l'administration de la ville à l'époque prussienne et de la période qui a suivi, jusqu'au national-socialisme. En font partie des volumes importants de l'époque où Konrad Adenauer était maire de la ville. Depuis la Seconde Guerre mondiale figure parmi les acquisitions le dépôt des différents services de l'administration urbaine. S'y ajoutent des plans et des legs d'architectes avec des croquis, d'autres plans et modèles et aussi des photos et toutes sortes d'autres choses qu'il serait trop long de détailler ici. Ce sont précisément les dépôts des époques les plus récentes qui constituaient le gros des documents conservés aux archives municipales.

Il faut globalement souligner qu'avant de s'effondrer, les archives étaient tenues avec ordre. À tout moment, les collègues pouvaient mettre à la disposition des chercheurs et des citadins qui y portaient de l'intérêt, les fonds répertoriés dans des registres. Contrairement à ce qui s'est dit ça et là, on ne peut absolument pas prétendre que, depuis quelque temps, les archives étaient laissées à l'abandon. Les archives ont toujours été un lieu dédié à la recherche, ne serait-ce que du fait que le latin, le dialecte local et l'écriture manuscrite des documents posaient des problèmes presque insurmontables pour la plupart des non-initiés, sans parler du simple fait que la compréhension de nombreux concepts de l'époque était problématique. "Archives des citadins », comme on peut lire ça et là, les archives historiques de Cologne ne l'étaient qu'à certaines conditions, car la plupart des «citadins » ne possédaient pas les compétences nécessaires pour déchiffrer les archives et ne les possèderont pas davantage à l'avenir.

Avec leurs trésors, les archives municipales de Cologne offraient toutefois aux chercheurs allemands et étrangers un champ d'action très riche dont témoigne le nombre important de publications dans de nombreuses langues du monde entier. Cet effondrement signifie pour la science historique en Allemagne et à l'étranger une perte douloureuse, au moins pour un certain temps. Il ne signifie toutefois pas la fin de tout travail de réflexion sur l'histoire de Cologne et les questions et problématiques liées à la ville, d'autant plus que de nombreuses sources ont été publiées et que l'on peut découvrir des documents en lien avec la ville dans les archives extérieures à Cologne, et qu'il y a en un certain sens un dépôt parallèle des sources. Dans quelques années, les fonds que l'on aura pu sauver des 
anciennes archives de Cologne seront à nouveau accessibles et à la disposition de la recherche, même si nous ne pouvons encore indiquer un délai.

Comme les registres ont été préservés, on devrait pouvoir évaluer la quantité des pertes subies. Nous en sommes toutefois encore très loin, et bien plutôt occupés à nettoyer les documents rescapés et à les trier sommairement. Il s'agit avant tout de rendre à nouveau accessibles et consultables les documents humides ou mouillés, ceci par le procédé de " séchage à froid ». Ce procédé prendra du temps et nous ne pouvons indiquer quel délai sera nécessaire. Trop d'éléments dépendent de la quantité de documents extraits des décombres, des capacités mises à notre disposition et d'autres impondérables. Ceci est valable également pour la mise ou la remise en ordre des archives épargnées. Il faudra identifier les documents et les répartir dans les sections correspondantes, afin de les répertorier dans les registres dont nous disposons ou dans ceux qui seront refaits. Ceci aussi prendra du temps, sans que l'on puisse préciser aujourd'hui combien. Non pas tous, mais certains documents ont été déchirés par la violence de la chute du plafond et d'autres parties du bâtiment. Une fois encore, cela demandera du temps avant que les pièces épargnées puissent être remises en ordre. Pour cela on a besoin d'expérience, de la connaissance des fonds et d'une agilité de lecture de la part de ceux à qui l'on confiera ce travail.

Dès maintenant sont disponibles non seulement les registres mais aussi les microfilms réalisés dans le cadre des sauvegardes de sécurité depuis de nombreuses années. Presque tous les fonds anciens ont été microfilmés et des copies de ces films sont disponibles. Les documents isolés qui ont été ajoutés avec le temps, parce qu'on a retrouvé ailleurs une partie des actes, sont absents des microfilms. Ceux-ci, de ce fait, ne sont pas toujours complets. Mais dans ce cas, il s'agit la plupart du temps d'exceptions de peu d'importance. De telles sauvegardes existent également pour les documents d'après 1814. Ce n'est pas le cas, en revanche, pour les dépôts transférés aux archives récemment, ainsi que pour une partie des legs et photos. Si on devait ne pas retrouver ces pièces, elles appartiendraient aux trésors irrémédiablement perdus des archives, pour lesquels il n'y a pas de documents de remplacement.

Le problème est que la consultation des microfilms nécessite qu'un espace équipé d'appareils de lecture soit mis à disposition. Pour le moment, il n'y en a pas. De plus, de nombreux films n'ont pas été établis avec suffisamment de soin pour que l'on puisse les consulter sans autre forme de procès. Dans le passé il était d'usage, dans le doute, de jeter un œil à l'original avant de se décider pour l'une ou l'autre version, notamment pour les premiers microfilms, réalisés par des entreprises privées. Sont également concernées les versions microfilmées des Schreinsbücher, un type de source évoqué plus haut, et qui faisait la singularité des anciennes archives de Cologne.

La numérisation des fonds avait commencé, la plupart des plans ont déjà été saisis. Cependant, l'entreprise n'en était encore qu'à ses débuts lorsque le bâtiment abritant les fonds s'est effondré. Une simple numérisation des films existants, comme cela est prévu, ne résout pas les problèmes mentionnés ci-dessus, car ce procédé ne peut remédier aux lacunes de ces supports. Toutefois, ces microfilms et leur numérisation seront à court terme les seuls documents disponibles pour des recherches à venir.

En ce qui concerne les pertes archivistiques, des chiffres sans cesse renouvelés sont donnés par les médias. Il est toutefois impossible de donner une estimation un tant soit peu fiable pour le moment. Même des chiffres approximatifs sont impossibles à fournir à l'heure actuelle, car les fonds ont été éparpillés, quelques volumes mêmes démembrés par 
le sinistre, et les documents rescapés n'ont pas été mis en regard des registres. Du reste, des documents que l'on croyait perdus sont encore extraits des décombres et mis à l'abri. Une vue d'ensemble des pertes assurément constatables ne sera possible que lorsqu'on aura entreposé les documents épargnés et restaurés dans un nouveau bâtiment et que l'on en aura contrôlé le nombre exact à l'aide des répertoires. D'ici là, il faudrait considérer avec une méfiance certaine mais justifiée tous les chiffres avancés.

En tant qu'ancien rapporteur scientifique des Archives Historiques de Cologne, je ne peux qu'espérer que l'on sauvera un maximum de documents et que l'on trouvera rapidement un emplacement pour construire un nouveau bâtiment et mettre ensuite les documents épargnés à la disposition des chercheurs. Car les documents sauvés et restaurés ont leur place dans la ville de Cologne et ne devraient pas, à long terme, disparaître dans différentes archives et bibliothèques. 